\title{
Efficacy of immune-checkpoint inhibitors in advanced non-small cell lung cancer patients with different metastases
}

\author{
Meng Qiao ${ }^{1}$, Fei Zhou ${ }^{1}$, Likun Hou ${ }^{2}$, Xuefei Li $^{3}$, Chao Zhao ${ }^{3}$, Tao Jiang', Guanghui Gao ${ }^{1}$, Chunxia Su ${ }^{1}$, \\ Chunyan $\mathrm{Wu}^{2 \#}$, Shengxiang Ren ${ }^{1 \#}$, Caicun Zhou ${ }^{1 \#}$ \\ ${ }^{1}$ Department of Medical Oncology, Shanghai Pulmonary Hospital, Tongji University School of Medicine, Shanghai, China; ${ }^{2}$ Department of \\ Pathology, Shanghai Pulmonary Hospital, Tongji University School of Medicine, Shanghai, China; ${ }^{3}$ Department of Lung Cancer and Immunology, \\ Shanghai Pulmonary Hospital, Tongji University School of Medicine, Shanghai, China \\ Contributions: (I) Conception and design: C Wu, S Ren, C Zhou; (II) Administrative support: None; (III) Provision of study materials or patients: \\ None; (IV) Collection and assembly of data: M Qiao, F Zhou, L Hou; (V) Data analysis and interpretation: X Li, C Zhao, T Jiang, G Gao, C Su; (VI) \\ Manuscript writing: All authors; (VII) Final approval of manuscript: All authors. \\ \#The authors contributed equally as senior authors. \\ Correspondence to: Prof. Caicun Zhou. Department of Medical Oncology, Shanghai Pulmonary Hospital \& Thoracic Cancer Institute, Tongji \\ University School of Medicine, No. 507, Zheng Min Road, Shanghai 200433, China. Email: caicunzhou_dr@163.com.
}

Background: To investigate the significance of metastatic sites and their numbers to the efficacy of immune checkpoint inhibitors (ICIs) in patients with advanced non-small cell lung cancer (NSCLC).

Methods: A total of 232 patients who received ICI monotherapy or ICI-based combination therapy were retrospectively identified from January 2016 to February 2019. Six metastatic sites (brain, liver, bone, adrenal gland, contralateral lung, pleura) were included to analyze their significance to ICI efficacy. To explore the association between liver metastasis (LM) and tumor $\mathrm{T}$ cell infiltration, 46 patients with available tumor specimens were tested for PD-L1 expression, CD8+ tumor infiltrating lymphocytes (TILs) density. Overall survival (OS) and progression-free survival (PFS) were estimated by Kaplan-Meier curves.

Results: More metastatic organs involved were associated with significantly worse PFS (0-1 organ: 5.7 months, $2-3$ organs: 3.5 months, $\geq 4$ organs: 2.7 months, $\mathrm{P}<0.001)$ and lower ORR (36\% vs. $29.8 \%$ vs. $18.2 \%, \mathrm{P}<0.001)$. Patients with brain metastasis $(\mathrm{BM})$ had shorter PFS and $\mathrm{OS}$ than those without $(\mathrm{P}=0.002$, $\mathrm{P}=0.021$; respectively). Notably, patients with LM had the shortest PFS (2.3 months, $\mathrm{P}=0.005)$ and numerically shortest OS (9.8 months, $\mathrm{P}=0.238$ ) compared with those with other organ metastases. Multivariate analysis revealed that $L M$ was independently associated with inferior PFS $(\mathrm{P}<0.001)$. Immunostaining showed that patients with $\mathrm{LM}$ tended to have lower proportions of PD-L1+CD8+TIL+ tumors compared with those without $\mathrm{LM}(0 \%$ vs. 30.8\%, P=0.088). Interestingly, ICI-based combination therapy could effectively control LM with improved intrahepatic PFS ( $\mathrm{P}=0.056)$ and ORR (41.7\% vs. 6.7\%, $\mathrm{P}=0.030)$.

Conclusions: More metastatic organs involved were associated with poorer response to ICIs. LM was a negative predictive factor for patients treated with ICI monotherapy and the combination strategy might effectively control LM.

Keywords: Non-small cell lung cancer (NSCLC); immune checkpoint inhibitors (ICIs); immunostaining; programmed-death 1 (PD-1); programmed death-ligand 1 (PD-L1); metastases

Submitted Feb 10, 2020. Accepted for publication Sep 30, 2020.

doi: 10.21037/atm-20-1471

View this article at: http://dx.doi.org/10.21037/atm-20-1471 


\section{Introduction}

Lung cancer is the leading cause of cancer-related deaths worldwide with high incidence of distant metastasis (1). Treatment targeting immune-checkpoints, including programmed-death 1 (PD-1)/its ligand (PD-L1) interaction, is a breakthrough and significantly prolongs the survival of patients with advanced non-small cell lung cancer (NSCLC), specifically, those without oncogenic drivers (2). However, metastasis is still unpreventable and incurable, and remains a main cause for cancer-related deaths. The most frequent metastatic sites in NSCLC are bone, brain, lung, liver, adrenal gland, and pleura (3). With the wide application of immune checkpoint inhibitors (ICIs), the efficacy of ICIs in patients with different metastatic sites needs to be further elucidated.

Few clinical trials have reported the subgroup data based on metastatic sites at baseline in patients treated with ICIs. As to brain metastasis (BM), the dismal results regarding the efficacy of ICI monotherapy in patients with CNS metastasis compared with chemotherapy have been reported in KEYNOTE-024, CheckMate-057 and OAK (4-6). However, promising results of pembrolizumab in combination with chemotherapy are noted in KEYNOTE-189 (7). In terms of liver metastasis (LM), minimal therapeutic benefit has been observed with the use of ICI monotherapy. Furthermore, combining atezolizumab (anti-PD-L1 antibody) with chemotherapy is also not an effective approach. Notably, the addition of bevacizumab to atezolizumab and chemotherapy appears to improve survival outcomes in patients with LM at baseline (8). Therefore, for patients with specific metastatic sites, the efficacy of ICIs is still unclear. Additionally, to our knowledge, no prospective studies focus on the efficacy of ICIs in patients with bone, contralateral lung, adrenal gland, and pleural metastases.

Herein, in this study, we aimed to investigate the significance of different metastatic sites and their numbers to the efficacy of ICIs in patients with advanced NSCLC.

We present the following article in accordance with the STROBE reporting checklist (available at http://dx.doi. org/10.21037/atm-20-1471).

\section{Methods}

The study was conducted in accordance with the Declaration of Helsinki (as revised in 2013) and was approved by the Ethics Committee of Shanghai Pulmonary Hospital, Tongji University School of Medicine (No. K18-
089-1) and individual consent for this retrospective analysis was waived.

\section{Patients}

As shown in Figure S1, patients who were diagnosed with advanced NSCLC between January 2016 and February 2019 and received ICIs during the treatment course with complete medical records (baseline radiological data and available follow-up) in Shanghai Pulmonary Hospital were enrolled in this retrospective study. Electronic medical records were collected including, age, sex, ECOG PS, smoking history, pathology, staging, driver mutations, ICI treatment strategy, and immunotherapy treatment line. In addition, to investigate the significance of metastatic sites to clinical outcome of ICIs, six metastatic sites, namely the brain, the liver, the bone, the adrenal gland, the contralateral lung, and the pleura were included in this present study. Patients were followed up until October 2019.

\section{Efficacy evaluation}

Progression-free survival (PFS) was defined from the first day of ICIs treatment to the day of physician assessment of disease progression or death from any cause. Overall survival (OS) was calculated from the date of ICI treatment start to the date of death of any cause or last follow-up. Objective response rate (ORR) was assessed by investigators according to the Response Evaluation Criteria In Solid Tumors (RECIST; version 1.1). ORR was calculated as percentage of patients with evaluated complete response (CR) or partial response (PR).

To detect the metastatic pattern, patients were assessed by chest CT scan, brain MRI, bone scan and abdominal ultrasound or PET-CT before application of ICIs.

\section{Immunobistochemistry and detection of oncogenic drivers}

The tumor specimens were fixed in formalin and embedded in paraffin in accordance with standardized procedures $(9,10)$. IHC staining, according to our previous study, was performed using PD-L1 IHC 22C3 pharmDx assay (DAKO) and CD8 monoclonal antibodies (\#M7103, clone C8/144B, DAKO, Glostrup, Denmark, diluted 1:200) to detect the expression of $\mathrm{PD}-\mathrm{L} 1$ and $\mathrm{CD} 8^{+}$tumor infiltrating lymphocytes (TILs) in specimens. PD-L1 expression 
on tumor cells was defined by tumor proportion scores (TPS) and the cut-off value was $1 \%$ (11). CD ${ }^{+}$TILs were determined as lymphocytes with cytoplasmic expression of CD8 infiltrating in the central or marginal tumor region and $\mathrm{CD} 8^{\text {low/high }}$ was defined by cut-off value of $5 \%$ $(9,12)$. IHC scores were independently determined by two experienced pathologists ( $\mathrm{L} \mathrm{Hou}, \mathrm{C} \mathrm{Wu}$ ).

The oncogenic driver mutation analysis was performed at Shanghai Pulmonary hospital (Shanghai, China). The analysis was performed using commercially available AmoyDx ${ }^{\circledR}$ Gene Mutation Detection Kits (AmoyDx Co. Ltd., Xiamen, China).

\section{Statistical analysis}

Baseline clinical characteristics were listed by numbers and percentages. To analyze the ORR difference between subgroups, the Chi-square or Fisher's exact test was performed. The Kaplan-Meier method was used to analyze the survival probability and log-rank test was used to calculate the significance of differences. Cox proportional hazard model was applied for the univariate and multivariate analyses to calculate the hazard rations (HR) and 95\% confidence intervals (95\% CI). Parameters with the univariate $\mathrm{P}$ value of less than 0.1 were included in multivariate model. All data were analyzed by using the Statistical Package for Social Science (SPSS) software (version 23.0 for Mac) and GraphPad Prism software (Version 6 for Mac). $\mathrm{P}$ values in this article were two-sided and considered statistically significant when less than 0.05 .

\section{Results}

\section{Baseline characteristics and efficacy of ICIs in total population}

Baseline characteristics are summarized in Table 1. A total of 232 advanced NSCLC patients were identified. The median age was 62 (20 to 82$)$ years old and $83.2 \%(193 / 232)$ were male. Among them, 62.9\% (146/232) of patients had smoking history and 62.9\% (146/232) were diagnosed as lung adenocarcinoma. 42 patients had genetic aberrations, of which $50 \%$ (21/42) harbored KRAS mutations and $33.3 \%$ (14/42) with EGFR mutations (Figure S2). 128 patients (55.2\%) received ICI monotherapy, 64 (27.6\%) received ICI plus chemotherapy and the remaining received ICI plus anti-angiogenesis therapy. Detailed regimens are listed in Table S1. Among 232 patients, 66 (28.4\%) were treated with ICI as first-line treatment strategy. In our cohort, $54.3 \%$ (126/232) patients had 0-1 metastatic organ involved before the application of ICIs, 36.2\% (84/232) of patients had 2-3 metastatic organs and the rest of patients suffered from at least 4 metastatic organs. In terms of metastatic sites, the baseline incidence of BM was $12.5 \%$ (29/232), LM was $12.5 \%$ (29/232), bone metastasis was $37.5 \%(87 / 232)$, adrenal metastasis was $12.5 \%$ (29/232), pleural metastasis was $31.9 \%(74 / 232)$ and contralateral lung metastasis was $48.7 \%(113 / 232)$. As shown in Figure $1 A$, the median PFS was 4.5 months, median OS was 19.7 months and ORR was $32.0 \%$ in overall population of patients.

\section{Predictive and prognostic factors including metastatic burden and metastatic sites}

We performed a univariate and multivariate analysis to determine the factors that were associated with the efficacy of ICIs. As shown in Tables 2,3, patients with good performance status or treated with first-line ICIs had better PFS and OS. In addition, patients treated with combination strategy could get PFS benefit (HR 0.583, $\mathrm{P}=0.001$ ) rather than OS benefit. Younger patients (age <65) could get marginally significant OS benefit rather than PFS benefit from ICIs (univariate: $\mathrm{HR} 1.526, \mathrm{P}=0.060$; multivariate: HR 1.593, $\mathrm{P}=0.050)$. With regard to metastatic burden, more metastatic organs involving were associated with shorter PFS in both univariate $(\mathrm{P}<0.001)$ and multivariate analysis $(\mathrm{P}=0.006)$ and worse $\mathrm{OS}(\mathrm{P}=0.039)$ in univariate analysis. In terms of metastatic sites, LM or BM was significantly associated with shorter PFS (LM: HR 2.429, $\mathrm{P}<0.001 ; \mathrm{BM}$ : HR 1.961, $\mathrm{P}=0.002$ ) and OS (LM: HR 1.875, $\mathrm{P}=0.034$; $\mathrm{BM}$ : HR 1.999, $\mathrm{P}=0.023$ ) in univariate analysis. However, in multivariate analysis, only LM was identified as an independently predictive factor for PFS (HR 1.789, $\mathrm{P}=0.014)$.

\section{Clinical outcomes by metastatic burden and metastatic site}

We further evaluated the association between number of metastatic organs and clinical outcome in patients treated with ICIs (Figure 1B). Three subgroups were categorized based on the number of metastatic organs: $0-1,2-3$ and $\geq 4$ organs involved. More metastatic organs involving were associated with inferior PFS and ORR (median PFS: 5.7 vs. 3.5 vs. 2.7 months, $\mathrm{P}<0.001$; ORR: 36\% vs. $29.8 \%$ 
Table 1 Patient characteristics

\begin{tabular}{|c|c|}
\hline Characteristic & No. of patients $(n=232)$ \\
\hline Median age, years (range) & 62 (20 to 82) \\
\hline \multicolumn{2}{|l|}{ Sex, n (\%) } \\
\hline Male & $193(83.2)$ \\
\hline Female & $39(16.8)$ \\
\hline \multicolumn{2}{|l|}{ ECOG PS, n (\%) } \\
\hline $0-1$ & $226(97.4)$ \\
\hline 2 & $6(2.6)$ \\
\hline \multicolumn{2}{|l|}{ Smoking history, n (\%) } \\
\hline Current/former & $146(62.9)$ \\
\hline Never & $86(37.1)$ \\
\hline \multicolumn{2}{|l|}{ Pathology, n (\%) } \\
\hline Adenocarcinoma & $146(62.9)$ \\
\hline Squamous & $64(27.6)$ \\
\hline Others & $22(9.5)$ \\
\hline \multicolumn{2}{|l|}{ Staging, n (\%) } \\
\hline IIIB-IV & $199(85.8)$ \\
\hline Post-operative recurrence & $33(14.2)$ \\
\hline \multicolumn{2}{|l|}{ Gene aberrations, n (\%) } \\
\hline Wild-type & $190(81.9)$ \\
\hline Mutated & $42(18.1)$ \\
\hline \multicolumn{2}{|l|}{ Treatment strategy, n (\%) } \\
\hline $\mathrm{ICl}$ alone & $128(55.2)$ \\
\hline $\mathrm{ICI}+$ chemotherapy & $64(27.6)$ \\
\hline $\mathrm{ICI}+$ anti-angiogenesis & $40(17.2)$ \\
\hline \multicolumn{2}{|l|}{ Treatment line, $\mathrm{n}(\%)$} \\
\hline First & $66(28.4)$ \\
\hline Second & $94(40.5)$ \\
\hline$\geq$ Third & $72(31.1)$ \\
\hline \multicolumn{2}{|c|}{ Number of metastatic sites, $\mathrm{n}(\%)$} \\
\hline $0-1$ & $126(54.3)$ \\
\hline $2-3$ & $84(36.2)$ \\
\hline$\geq 4$ & $22(9.5)$ \\
\hline \multicolumn{2}{|l|}{ Brain metastasis, $\mathrm{n}(\%)$} \\
\hline Yes & $29(12.5)$ \\
\hline No & $203(87.5)$ \\
\hline
\end{tabular}

Table 1 (continued)
Table 1 (continued)

\begin{tabular}{lc}
\hline Characteristic & No. of patients $(\mathrm{n}=232)$ \\
\hline Liver metastasis, $\mathrm{n}(\%)$ & $29(12.5)$ \\
Yes & $203(87.5)$ \\
No & \\
Bone metastasis, $\mathrm{n}(\%)$ & $87(37.5)$ \\
Yes & $145(62.5)$ \\
No & \\
Adrenal metastasis, $\mathrm{n}(\%)$ & $29(12.5)$ \\
Yes & $203(87.5)$ \\
No & \\
Pleural metastasis, $\mathrm{n}(\%)$ & $74(31.9)$ \\
Yes & $158(68.1)$ \\
No & \\
Contralateral lung metastasis, $\mathrm{n}(\%)$ & $113(48.7)$ \\
Yes & $119(51.3)$ \\
No & \\
\hline & \\
\hline
\end{tabular}

vs. $18.2 \%, \mathrm{P}<0.001)$. Moreover, patients with at least 4 metastatic organs involved had the shortest OS, whereas OS was not statistically different between patients with $0-1$ and 2-3 metastatic organs due to the limited sample size (median OS: 23.5 vs. 23.8 vs. 9.9 months, $\mathrm{P}=0.073$ ).

We next investigated the significance of distinct metastatic sites on the efficacy of ICIs, as shown in Figure $1 C$, patients with LM had significantly shortest PFS compared with those with other metastatic patterns (LM: 2.3 months, $\mathrm{P}=0.005$ ) and numerically shortest $\mathrm{OS}$ (LM: 9.8 months), though the statistical significance was not reached $(\mathrm{P}=0.238)$. Additionally, as presented in Figure 2, the presence of $\mathrm{LM}$ or $\mathrm{BM}$ rather than other metastatic organs was associated with shorter PFS (LM: 2.3 vs. 5.0 months, $\mathrm{P}<0.001$; $\mathrm{BM}: 3.0$ vs. 4.7 months, $\mathrm{P}=0.002)$ and $\mathrm{OS}$ (LM: 9.8 vs. 23.5 months, $\mathrm{P}=0.031$; $\mathrm{BM}$ : 9.9 vs. 23.5 months, $\mathrm{P}=0.021)$.

Having identified the significance of metastatic site to response, we examined the relationship between different metastatic sites and clinical outcomes in monotherapy group and combination therapy group, respectively (Figures S3,S4). The presence of LM was associated with inferior PFS in both monotherapy and combination treatment group (median PFS: monotherapy group: 2.0 
A
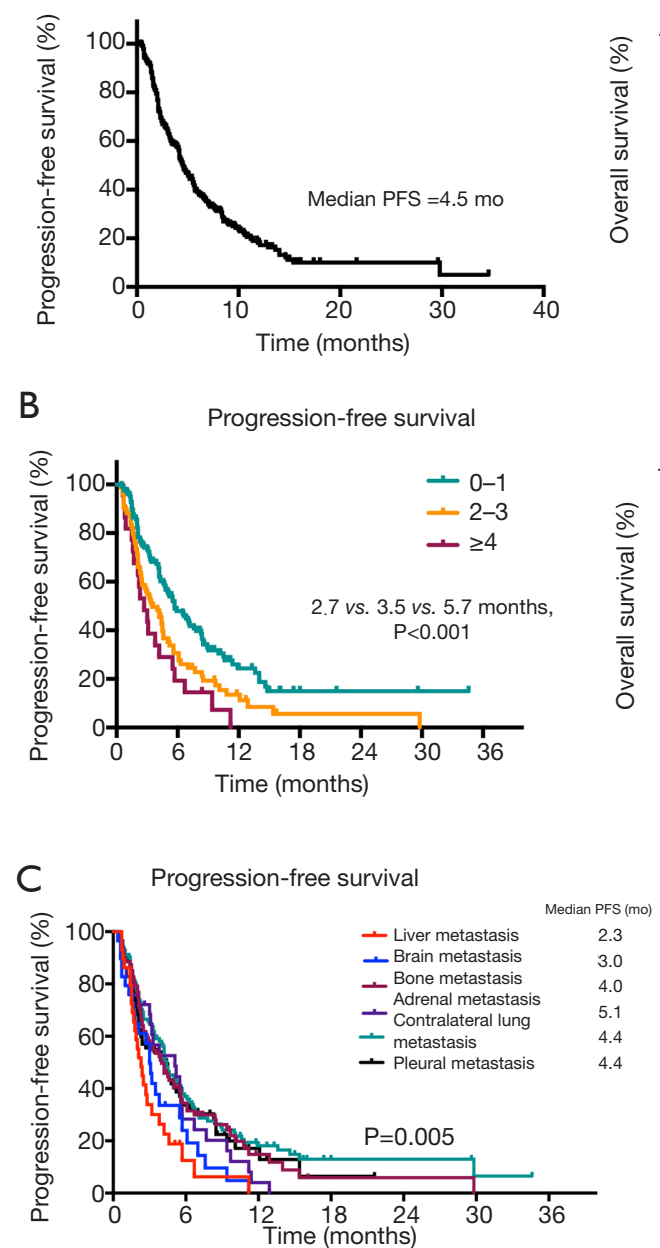

Overall survival

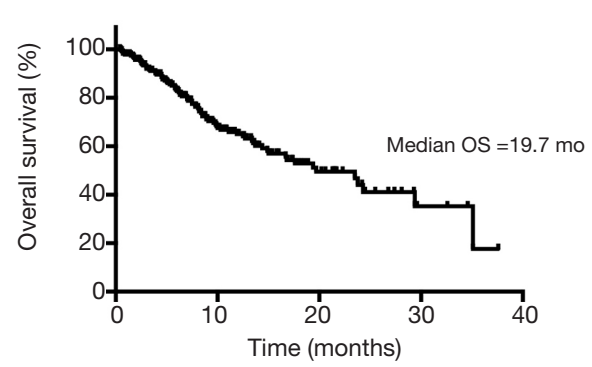

Overall survival

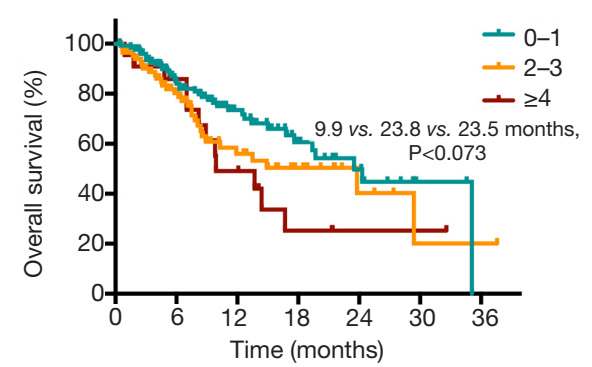

Objective response

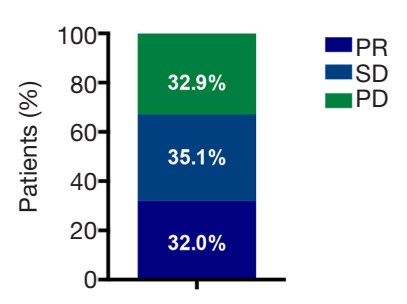

Objective response

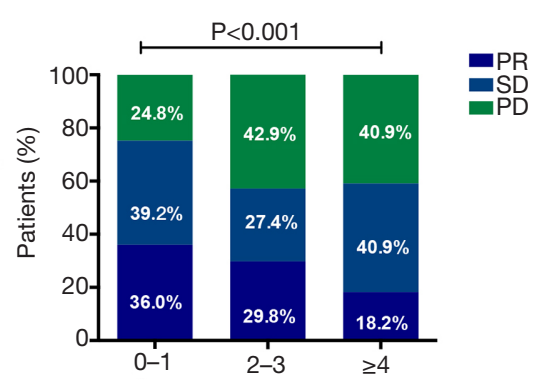

Figure 1 PFS, OS and objective response in patients treated with ICI (A) in overall cohort; (B) stratified by numbers of metastatic organs; (C) stratified by metastatic sites. ICI, immune checkpoint inhibitor.

vs. 3.2 months, $\mathrm{P}=0.010$; combination treatment group: 3.2 vs. 7.0 months, $\mathrm{P}<0.001)$ compared with those without LM. Patients with BM had shorter PFS in monotherapy group but not in combination treatment group than those without BM (median PFS: monotherapy cohort: 1.7 vs. 3.3 months, $\mathrm{P}<0.001)$. For other metastatic sites, the presence of metastasis was not associated with the efficacy of ICIs except for the presence of bone metastasis (median PFS: 2.3 vs. 3.4 months, $\mathrm{P}=0.04$ ) or pleural metastasis (median PFS: 2.1 vs. 3.4 months, $\mathrm{P}=0.024$ ) in monotherapy group. For OS, patients with BM had inferior OS than those without in combination treatment group (median OS: 13.7 vs. NA, $\mathrm{P}=0.028$ ) and patients with pleural metastasis had shortened OS (median OS: 8.2 vs. 17.6 months, $\mathrm{P}=0.045$ ) than those without in monotherapy group.

\section{Association of LM with TME subtypes}

Given the negative value of LM on ICI efficacy, we investigated association of T-cell infiltrates and LM with IHC analysis of CD8 and PD-L1 expression in samples obtained from 46 patients with enough tissue before treatment. Detailed clinical characteristics of 46 patients are shown in Table S2. 7 of 46 patients had baseline LM. There were no significant differences observed with regard to age $(\mathrm{P}=0.717)$, gender $(\mathrm{P}=1.000)$, smoking status ( $\mathrm{P}=0.313)$, histology $(\mathrm{P}=0.626)$, staging $(\mathrm{P}=0.496)$, genetic aberration $(\mathrm{P}=0.941)$ and treatment strategy $(\mathrm{P}=0.698)$ by LM status. PD-L1 positive tumors were detected in $14.3 \%$ (1/7) patients with LM and 38.5\% (15/39) without LM. Positive CD8 ${ }^{+}$TILs were detected in $71.4 \%(5 / 7)$ patients 
Table 2 Univariate and multivariate analyses of clinical parameters on PFS in patients treated with ICIs

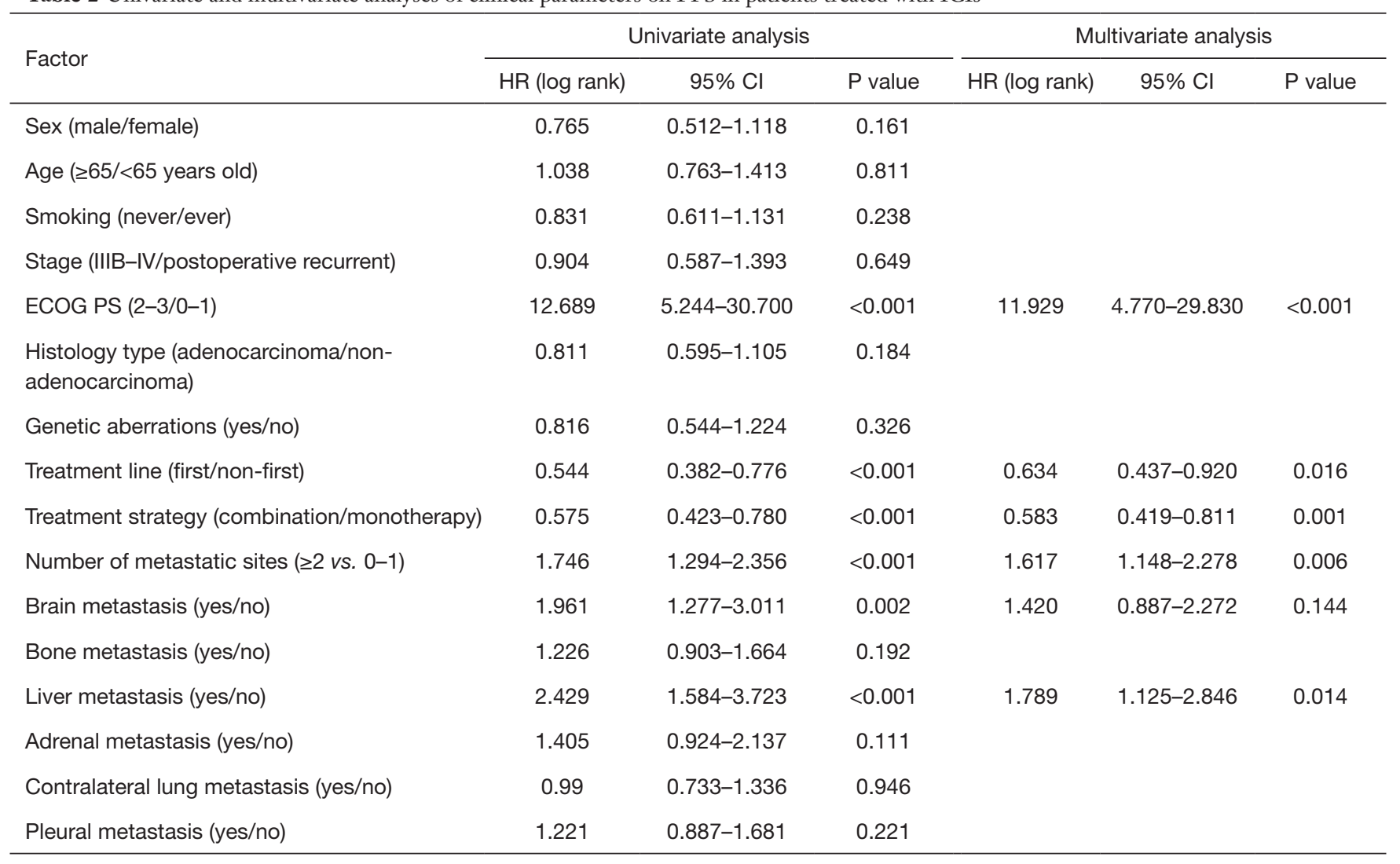

with LM and $79.5 \%(31 / 39)$ without LM. No association was observed in PD-L1 or CD8 ${ }^{+}$expression and LM status ( $\mathrm{P}=0.216$ and $\mathrm{P}=0.634$, respectively).

Next, based on PD-L1 expression and $\mathrm{CD} 8^{+} \mathrm{T}$ cell infiltration (13), the TME were categorized into four subtypes: $\mathrm{PD}-\mathrm{L}^{+} / \mathrm{CD}^{+}{ }^{+} \mathrm{TILs}^{+}(\mathrm{I}), \mathrm{PD}-\mathrm{L}^{-} / \mathrm{CD}^{+}{ }^{+} \mathrm{TILs}^{-}$ (II), $\mathrm{PD}-\mathrm{L}^{+} / \mathrm{CD}^{+}{ }^{+} \mathrm{TILs}^{-}$(III), PD-L1 ${ }^{-} / \mathrm{CD}^{+}{ }^{+} \mathrm{TILs}^{+}$(IV) (Figure $3 A$ ). In patients with $\mathrm{LM}$, the proportions of four TME subtypes were $0 \%$ (type I), 71.4\% (type IV), $14.3 \%$ (type II), and $14.3 \%$ (type III), respectively. In patients without LM, the proportions of four TME subtypes were $30.8 \%$ (type I), $48.7 \%$ (type IV), $7.7 \%$ (type II), and $12.8 \%$ (type III), respectively, suggesting that patients without LM tended to have higher proportions of $\mathrm{CD} 8^{+} \mathrm{PD}-\mathrm{L} 1^{+}$tumors compared with those with LM (30.8\% vs. $0 \%, \mathrm{P}=0.088)$ (Figure 3B).

\section{Efficacy of different ICI regimen in patients with LM}

Next, we explored the optimal therapeutic regimen for patients with LM. As shown in Figure 4A, patients who received ICI-based combination therapy had marginally longer PFS and numerically longer OS than those treated with monotherapy (median PFS: 3.2 vs. 2.0 months, $\mathrm{P}=0.066$; median OS: 10.3 vs. 8.5 months, $\mathrm{P}=0.230$ ). ORR was also significantly increased in combination treatment group ( $45.5 \%$ vs. $6.3 \%, \mathrm{P}=0.033$ ). Additionally, combination therapy could effectively control LM with improved intrahepatic PFS $(\mathrm{P}=0.056)$ and intrahepatic ORR (41.7\% vs. $6.7 \%, \mathrm{P}=0.030$ ) (Figure $4 B$ ). Two representative cases were shown in Figure $\mathrm{S} 5$ and indicated that the addition of conventional therapy could effectively control LM.

\section{Discussion}

In this study, we investigated the significance of metastatic sites to the efficacy of ICIs in patients with advanced NSCLC. With more metastatic organs involved, worse clinical outcomes were observed. The presence of BM or LM was associated with shorter PFS and OS. Multivariate analysis showed that among all types of metastatic sites, LM was the only negative predictor for the efficacy of 
Table 3 Univariate and multivariate analyses of clinical parameters on OS in patients treated with ICIs

\begin{tabular}{|c|c|c|c|c|c|c|}
\hline Factor & \multicolumn{3}{|c|}{ Univariate analysis } & \multicolumn{3}{|c|}{ Multivariate analysis } \\
\hline Sex (male/female) & 1.173 & $0.635-2.170$ & 0.610 & & & \\
\hline Age $(\geq 65 /<65$ years old $)$ & 1.526 & $0.983-2.369$ & 0.060 & 1.593 & $1001-2.535$ & 0.050 \\
\hline Smoking (never/ever) & 1.116 & $0.702-1.774$ & 0.644 & & & \\
\hline ECOG PS (2-3/0-1) & 25.572 & $7.821-83.608$ & $<0.001$ & 24.173 & $7.092-82.392$ & $<0.001$ \\
\hline $\begin{array}{l}\text { Histology type (adenocarcinoma/non- } \\
\text { adenocarcinoma) }\end{array}$ & 0.741 & $0.476-1.154$ & 0.185 & & & \\
\hline Genetic aberrations (yes/no) & 0.956 & $0.517-1.770$ & 0.886 & & & \\
\hline Brain metastasis (yes/no) & 1.999 & $1.101-3.631$ & 0.023 & 1.676 & $0.844-3.330$ & 0.140 \\
\hline Bone metastasis (yes/no) & 1.260 & $0.813-1.955$ & 0.302 & & & \\
\hline Liver metastasis (yes/no) & 1.875 & $1.050-3.347$ & 0.034 & 1.191 & $0.604-2.351$ & 0.614 \\
\hline Adrenal metastasis (yes/no) & 1.274 & $0.689-2.358$ & 0.440 & & & \\
\hline Contralateral lung metastasis (yes/no) & 0.904 & $0.582-1.405$ & 0.654 & & & \\
\hline Pleural metastasis (yes/no) & 1.387 & $0.886-2.171$ & 0.152 & & & \\
\hline
\end{tabular}

PFS, progression-free survival; OS, overall survival; ECOG PS, Eastern Cooperation Oncology Group performance status.

ICIs. Patients with LM tended to have lower proportions of inflamed TME (PD-L1 $\left.{ }^{+} \mathrm{CD}^{+} \mathrm{TIL}^{+}\right)$tumors compared with those without LM. ICI combination treatment might effectively control liver progression compared with ICI monotherapy.

The bone, brain, liver, adrenal gland, contralateral lung and pleura were common metastatic sites in advanced NSCLC (14). Metastatic spread is the signal of advancedstage cancer and the major reason for cancer-related deaths (15). The number of metastatic organs often indicates the systemic tumor burden. Our study suggested that more metastatic organs involved were correlated with worse PFS, OS and ORR. Consistent with our findings, previous study reported that in ALK-positive NSCLC, the increasing number of metastatic organs were also associated with worse OS (16). Moreover, our study indicated that younger patients with good performance status and patients who received first-line ICIs could obtain OS benefit from the treatment. Immunotherapy, especially ICI was proposed to release the "brake" of $\mathrm{T}$ cells to produce the robust immune response. Owing to the memory function of $\mathrm{T}$ cells, a subset of patients who received immunotherapy usually demonstrated a tail of survival curve indicating the long-term benefit. However, the premise of the effective work was based on the comparatively complete immune system and good physical condition (17). Patients with low metastatic burden was associated with early phase of advanced NSCLC. Therefore, the immunotherapy should be applied as earlier as possible in patients with low metastatic burden, immune system that could be reinvigorated.

In the present study, patients with LM was associated with inferior response and PFS. LM was a negative prognostic factor in NSCLC patients. A population-based data on metastatic site performed by Riihimaki et al. reported that LM was associated with worst survival with an OS of only 3 months (3). Previous studies demonstrated that LM was associated with poor PFS in patients treated with nivolumab and pembrolizumab $(18,19)$. Reduced response in patients with LM could be partially explained as follows. (I) Owing 


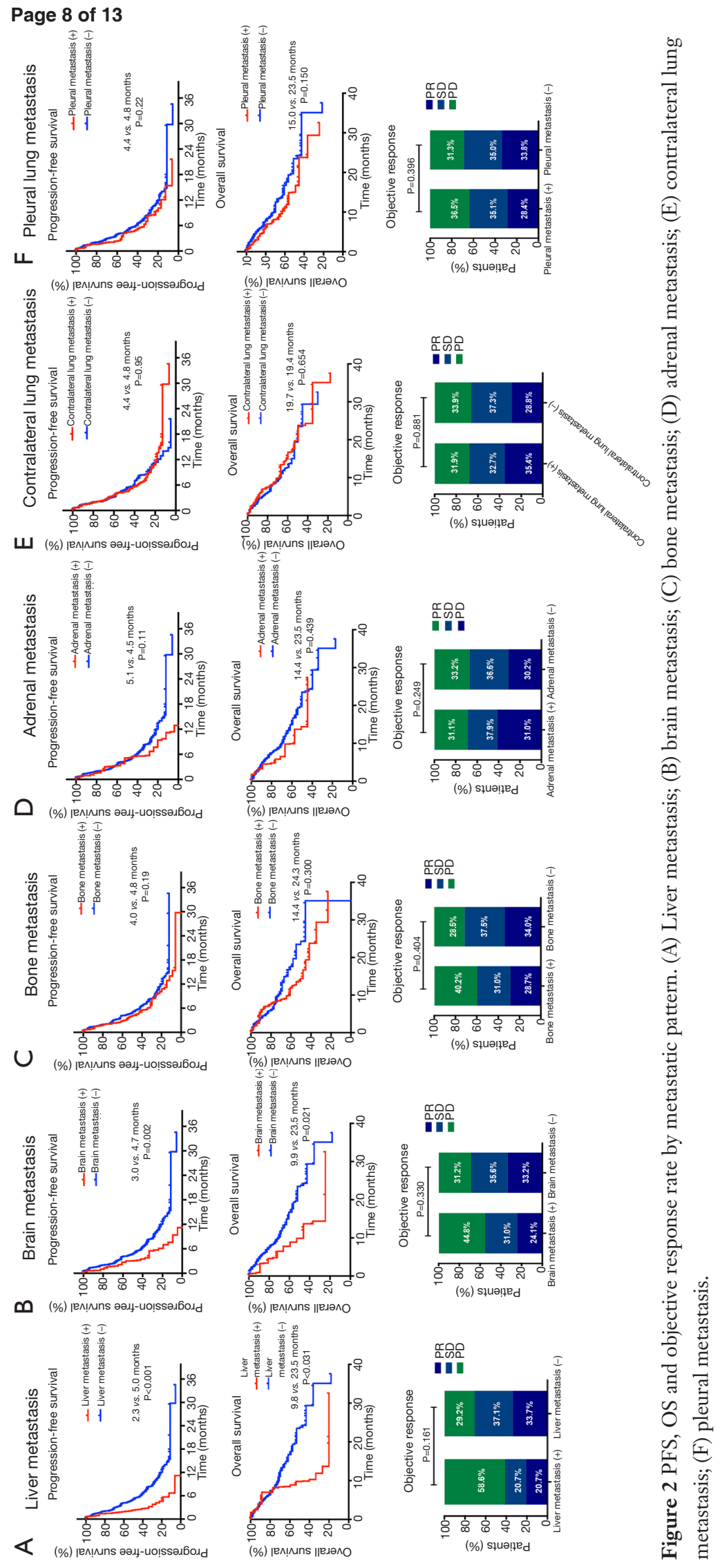

Page 8 of 13

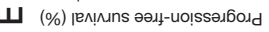

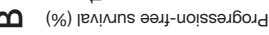

Qiao et al. Different metastases and $\mathrm{ICI}$ response

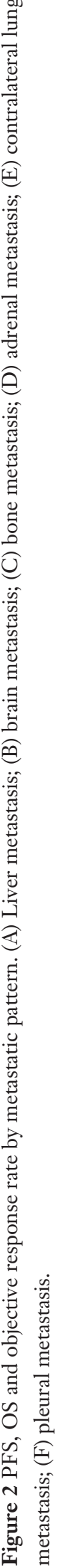


A I
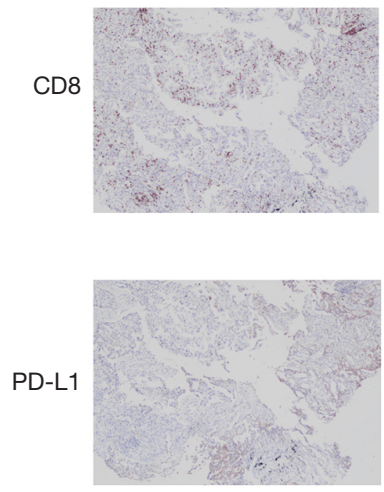

II
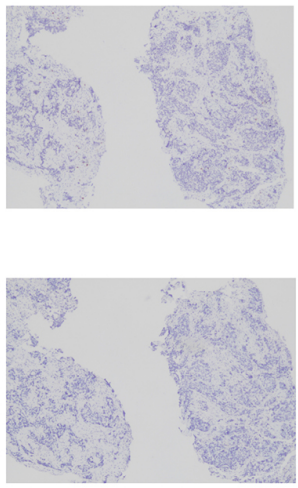

III
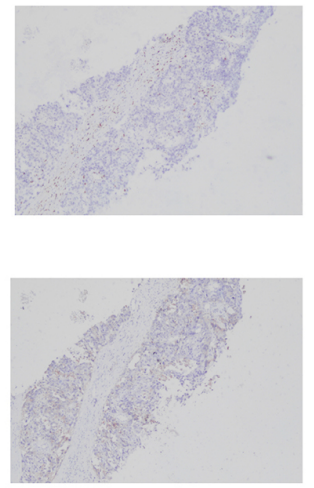

IV
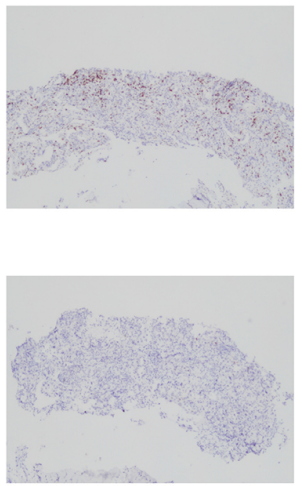

B

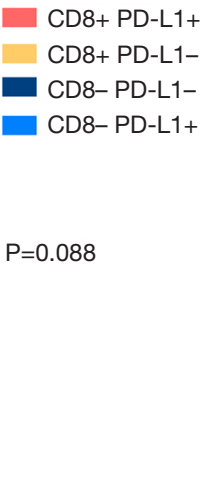

Figure 3 Association of LM and TME subtypes. (A) Representative immunohistochemistry images ( $\times 40$ ) of four TME subtypes with PD-L1 and CD8 expression; (B) proportions of 4 TME subtypes in patients with or without LM. TME, tumor microenvironment; LM, liver metastasis.

to liver-induced peripheral tolerance effect to maintain local immune microenvironment (20), patients with LM was not the beneficial population in immunotherapy setting. Liver-induced tolerance was firstly described in liver transplantation with the phenomenon that liver allografts were often without the need of histocompatibility (21). Previous studies pointed out several mechanisms that could account for liver-induced tolerance, including incomplete activation of $\mathrm{CD} 8^{+} \mathrm{T}$ cells, passive or active mechanisms trapping activated $\mathrm{CD} 8^{+} \mathrm{T}$ cells and activation of regulatory $\mathrm{T}$ cells $(22,23)$. (II) $\mathrm{LM}$ from lung cancer was reported to respond to treatment in a more similar way to liver cancer than lung cancer, in other word, in a way of tissue-specific immuno-regulation. Therefore, a hypoxia tumor condition in liver TME with high VEGF expression could contribute to the induction of immunosuppressive immune cells to form an immunosuppressive TME (24,25). (III) Reduced marginal $\mathrm{CD}^{+} \mathrm{T}$ cell infiltration was observed in liver (19). Poor TILs infiltration led to the ineffective response to immunotherapy. In our study, patients with LM tended to have lower proportions of Type I tumors which are regarded as likely to benefit from single-agent PD-1/PDL1 blockade (13). However, compared with chemotherapy, the pooled results showed that immunotherapy did benefit patients with baseline LM (HR for OS: $0.72, \mathrm{P}=0.008$, Figure S6). Of note, the included studies were regarding the comparison between ICI-based combination treatment and chemotherapy. Although after 3 years follow-up, nivolumab monotherapy demonstrated OS benefits versus docetaxel (26), there was no direct comparison between combination therapy and monotherapy, we firstly discovered that the combination of ICI with conventional treatment could control LM progression with significant improvement in intrahepatic PFS and ORR. The application of chemotherapy or anti-angiogenesis might alter the microenvironment in liver from tolerogenic to immunologic status. Since clinicians have multiple options to combine with ICI, which one is optimal? 
A
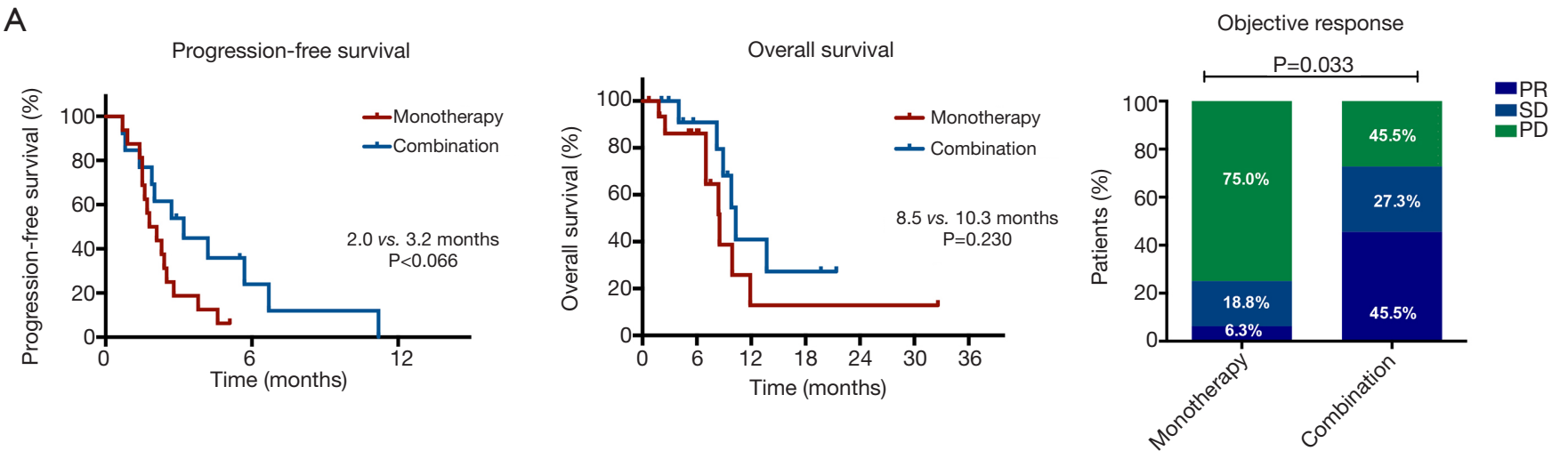

B
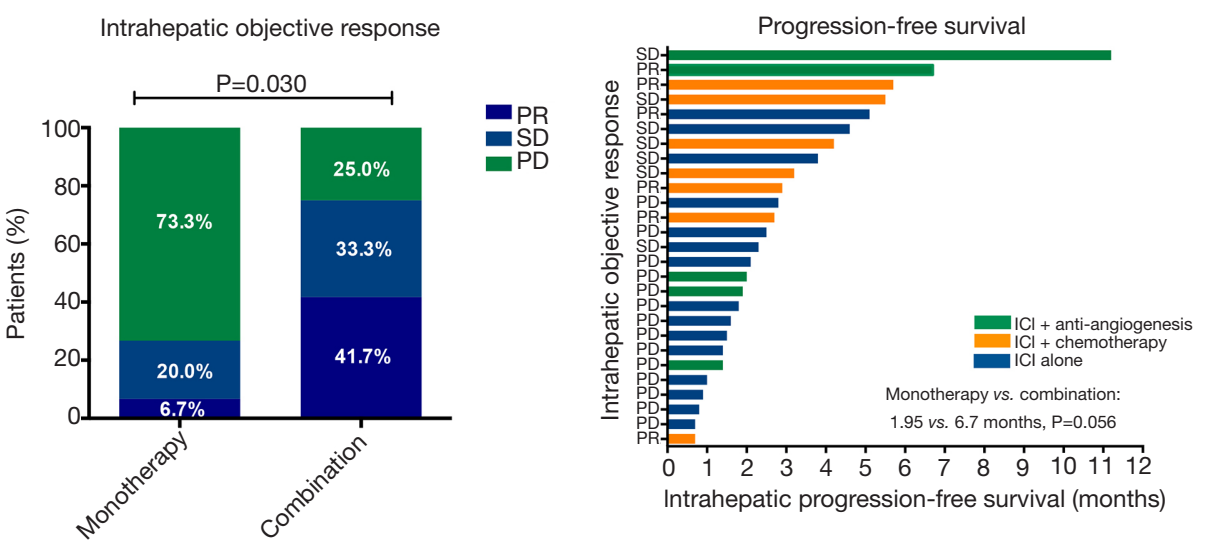

Figure 4 Combination treatment versus monotherapy in patients with LM. (A) PFS, OS and objective response; (B) the intrahepatic ORR and PFS. LM, liver metastasis; ORR, objective response rate; PFS, progression-free survival.

In metastatic liver microenvironment, the communications and interactions between tumor cells and resident cells, such as sinusoidal endothelium, stellate, Kupffer, and inflammatory cells supported tumor growth, angiogenesis, and mediated immunosuppression. Particularly, Kupffer cells produced cytokines and growth factors, such as VEGF, HGF and MMP to promote tumor cell proliferation and angiogenesis (27). The results from IMpower150 illustrated that three-agent combo (atezolizumab, bevacizumab, chemotherapy) seemed to have maximum effect in patients with LM. This result indicated the anti-angiogenesis might be important in controlling LM. Notably, the promising results from KEYNOTE-189 also showed that even twoagent (pembrolizumab and chemotherapy) combo also prolonged OS in patients with LM $(7,28)$. However, dismal results were shown in subgroup analysis from IMpower132 and IMpower150. Therefore, is anti-angiogenesis agent necessary in this setting? or did PD-1 or PD-L1 inhibitors have different impact in patients with LM? The treatment modality needs further investigation and validation.
$\mathrm{BM}$ is a tough and challenging case in the treatment of lung cancer together with poor prognosis. Totally, 25$30 \%$ of NSCLC patients were present with BM at initial diagnosis $(29,30)$. Since whole-brain radiotherapy (WBRT) for patients with BM can impair long-term memory and small BM cannot be controlled by stereotactic radiosurgery (SRS), novel systemic treatment for patients with BM are warranted (31). Of note, most clinical trials with regard to ICIs initially excluded or included fewer patients with BM and mostly, asymptomatic BM (32). Although univariate analysis and Kaplan-Meier curve showed that patients with BM had inferior PFS and OS compared with those without BM, multivariate analysis showed that BM was not associated with poorer survival. This finding was consistent with a recent study performed by Hendriks et al, after adjustment for number of metastatic organs and the use of corticosteroid in multivariate study, which were associated with poor PFS and OS (33). Therefore, patients with BM with no requirement for the use of corticosteroids and few metastatic organs could possibly get long-term survival 
benefits from ICIs and should not be simply excluded from clinical trials.

The study has several limitations. Firstly, given the nature of the retrospective study, the results need to be interpreted with caution. Secondly, PD-L1 testing is not mandatory when ICI was applied in the second or subsequent line of treatment. Therefore, PD-L1 expression status could not be included as confounding factors in our analyses. Additionally, the sample size for IHC is relatively small, only 46 patients enrolled including 7 with LM. Moreover, matched samples from LM to illustrate the liver TME might provide a more solid conclusion to distinguish the TME difference between patients with LM or without.

\section{Conclusions}

This study analyzed the significance of metastatic series to the efficacy of ICIs in one of the largest cohorts. Patients with more metastatic organs had inferior clinical outcomes. Multivariate analysis showed that BM was not associated with poor survival and LM was a negative signal in predicting the efficacy of ICI with lower proportions of inflamed TME. ICI combination therapy might effectively control liver progression over monotherapy. Although metastatic sites had an impact on the efficacy of ICIs, the potential benefit population and biomarker-guided in decision made still need more investigations in the era of precision medicine.

\section{Acknowledgments}

Funding: This work was supported by grants from the National Natural Science Foundation of China (No. 81672286, No. 81874036, No. 81703020), National R\&D projects (2016YFC0902300), Shanghai Science and Technology Medical Guidance Project (16411964400), Shanghai Innovative Collaboration Project (2020CXJQ02), Shanghai Science and Technology Innovative Project (19411950300).

\section{Footnote}

Reporting Checklist: The authors have completed the STROBE reporting checklist. Available at http://dx.doi. org/10.21037/atm-20-1471

Data Sharing Statement: Available at http://dx.doi. org/10.21037/atm-20-1471
Peer Review File: Available at http://dx.doi.org/10.21037/ atm-20-1471

Conflicts of Interest: All authors have completed the ICMJE uniform disclosure form (available at http://dx.doi. org/10.21037/atm-20-1471). The authors have no conflicts of interest to declare.

Etbical Statement: The authors are accountable for all aspects of the work in ensuring that questions related to the accuracy or integrity of any part of the work are appropriately investigated and resolved. The study was conducted in accordance with the Declaration of Helsinki (as revised in 2013) and was approved by the Ethics Committee of Shanghai Pulmonary Hospital, Tongii University School of Medicine (No. K18-089-1) and individual consent for this retrospective analysis was waived.

Open Access Statement: This is an Open Access article distributed in accordance with the Creative Commons Attribution-NonCommercial-NoDerivs 4.0 International License (CC BY-NC-ND 4.0), which permits the noncommercial replication and distribution of the article with the strict proviso that no changes or edits are made and the original work is properly cited (including links to both the formal publication through the relevant DOI and the license). See: https://creativecommons.org/licenses/by-nc-nd/4.0/.

\section{References}

1. Bray F, Ferlay J, Soerjomataram I, et al. Global cancer statistics 2018: GLOBOCAN estimates of incidence and mortality worldwide for 36 cancers in 185 countries. CA Cancer J Clin 2018;68:394-424.

2. Ribas A. Releasing the Brakes on Cancer Immunotherapy. N Engl J Med 2015;373:1490-2.

3. Riihimaki M, Hemminki A, Fallah M, et al. Metastatic sites and survival in lung cancer. Lung Cancer 2014;86:78-84.

4. Reck M, Rodriguez-Abreu D, Robinson AG, et al. Pembrolizumab versus Chemotherapy for PD-L1Positive Non-Small-Cell Lung Cancer. N Engl J Med 2016;375:1823-33.

5. Borghaei H, Paz-Ares L, Horn L, et al. Nivolumab versus Docetaxel in Advanced Nonsquamous Non-Small-Cell Lung Cancer. N Engl J Med 2015;373:1627-39.

6. Rittmeyer A, Barlesi F, Waterkamp D, et al. Atezolizumab versus docetaxel in patients with previously treated non-small-cell lung cancer (OAK): a phase 3, open- 
label, multicentre randomised controlled trial. Lancet 2017;389:255-65.

7. Gandhi L, Rodriguez-Abreu D, Gadgeel S, et al. Pembrolizumab plus Chemotherapy in Metastatic NonSmall-Cell Lung Cancer. N Engl J Med 2018;378:2078-92.

8. Reck M, Mok TSK, Nishio M, et al. Atezolizumab plus bevacizumab and chemotherapy in non-small-cell lung cancer (IMpower150): key subgroup analyses of patients with EGFR mutations or baseline liver metastases in a randomised, open-label phase 3 trial. Lancet Respir Med 2019;7:387-401.

9. Jiang T, Shi J, Dong Z, et al. Genomic landscape and its correlations with tumor mutational burden, PD-L1 expression, and immune cells infiltration in Chinese lung squamous cell carcinoma. J Hematol Oncol 2019;12:75.

10. Yang H, Shi J, Lin D, et al. Prognostic value of PD-L1 expression in combination with CD8(+) TILs density in patients with surgically resected non-small cell lung cancer. Cancer Med 2018;7:32-45.

11. Roach C, Zhang N, Corigliano E, et al. Development of a Companion Diagnostic PD-L1 Immunohistochemistry Assay for Pembrolizumab Therapy in Non-Small-cell Lung Cancer. Appl Immunohistochem Mol Morphol 2016;24:392-7.

12. Tokito T, Azuma K, Kawahara A, et al. Predictive relevance of PD-L1 expression combined with CD8+ TIL density in stage III non-small cell lung cancer patients receiving concurrent chemoradiotherapy. Eur J Cancer 2016;55:7-14.

13. Teng MW, Ngiow SF, Ribas A, et al. Classifying Cancers Based on T-cell Infiltration and PD-L1. Cancer Res 2015;75:2139-45.

14. Hess KR, Varadhachary GR, Taylor SH, et al. Metastatic patterns in adenocarcinoma. Cancer 2006;106:1624-33.

15. Turajlic S, Swanton C. Metastasis as an evolutionary process. Science 2016;352:169-75.

16. Pacheco JM, Gao D, Smith D, et al. Natural History and Factors Associated with Overall Survival in Stage IV ALKRearranged Non-Small Cell Lung Cancer. J Thorac Oncol 2019;14:691-700.

17. Blumenthal GM, Zhang L, Zhang H, et al. Milestone Analyses of Immune Checkpoint Inhibitors, Targeted Therapy, and Conventional Therapy in Metastatic NonSmall Cell Lung Cancer Trials: A Meta-analysis. JAMA Oncol 2017;3:e171029.

18. Shiroyama T, Suzuki H, Tamiya M, et al. Clinical
Characteristics of Liver Metastasis in Nivolumab-treated Patients with Non-small Cell Lung Cancer. Anticancer Res 2018;38:4723-9.

19. Tumeh PC, Hellmann MD, Hamid O, et al. Liver Metastasis and Treatment Outcome with Anti-PD-1 Monoclonal Antibody in Patients with Melanoma and NSCLC. Cancer Immunol Res 2017;5:417-24.

20. Horst AK, Neumann K, Diehl L, et al. Modulation of liver tolerance by conventional and nonconventional antigenpresenting cells and regulatory immune cells. Cell Mol Immunol 2016;13:277-92.

21. Jenne CN, Kubes P. Immune surveillance by the liver. Nat Immunol 2013;14:996-1006.

22. John B, Crispe IN. Passive and active mechanisms trap activated CD8+ T cells in the liver. J Immunol 2004;172:5222-9.

23. Crispe IN. Hepatic T cells and liver tolerance. Nat Rev Immunol 2003;3:51-62.

24. Chiu DK, Xu IM, Lai RK, et al. Hypoxia induces myeloidderived suppressor cell recruitment to hepatocellular carcinoma through chemokine (C-C motif) ligand 26. Hepatology 2016;64:797-813.

25. Facciabene A, Peng X, Hagemann IS, et al. Tumour hypoxia promotes tolerance and angiogenesis via CCL28 and T(reg) cells. Nature 2011;475:226-30.

26. Vokes EE, Ready N, Felip E, et al. Nivolumab versus docetaxel in previously treated advanced non-small-cell lung cancer (CheckMate 017 and CheckMate 057): 3-year update and outcomes in patients with liver metastases. Ann Oncol 2018;29:959-65.

27. Brodt P. Role of the Microenvironment in Liver Metastasis: From Pre- to Prometastatic Niches. Clin Cancer Res 2016;22:5971-82.

28. Socinski MA, Jotte RM, Cappuzzo F, et al. Atezolizumab for First-Line Treatment of Metastatic Nonsquamous NSCLC. N Engl J Med 2018;378:2288-301.

29. Barnholtz-Sloan JS, Sloan AE, Davis FG, et al. Incidence proportions of brain metastases in patients diagnosed (1973 to 2001) in the Metropolitan Detroit Cancer Surveillance System. J Clin Oncol 2004;22:2865-72.

30. Mujoomdar A, Austin JH, Malhotra R, et al. Clinical predictors of metastatic disease to the brain from non-small cell lung carcinoma: primary tumor size, cell type, and lymph node metastases. Radiology 2007;242:882-8.

31. Rosell R, Karachaliou N. Trends in immunotherapy for brain metastases. Lancet Oncol 2016;17:859-60. 
32. Harary M, Reardon DA, Iorgulescu JB. Efficacy and safety of immune checkpoint blockade for brain metastases. CNS Oncol 2019;8:CNS33.

33. Hendriks LEL, Henon C, Auclin E, et al. Outcome of

Cite this article as: Qiao $M$, Zhou F, Hou L, Li X, Zhao C, Jiang T, Gao G, Su C, Wu C, Ren S, Zhou C. Efficacy of immune-checkpoint inhibitors in advanced non-small cell lung cancer patients with different metastases. Ann Transl Med 2021;9(1):34. doi: 10.21037/atm-20-1471
Patients with Non-Small Cell Lung Cancer and Brain Metastases Treated with Checkpoint Inhibitors. J Thorac Oncol 2019;14:1244-54. 


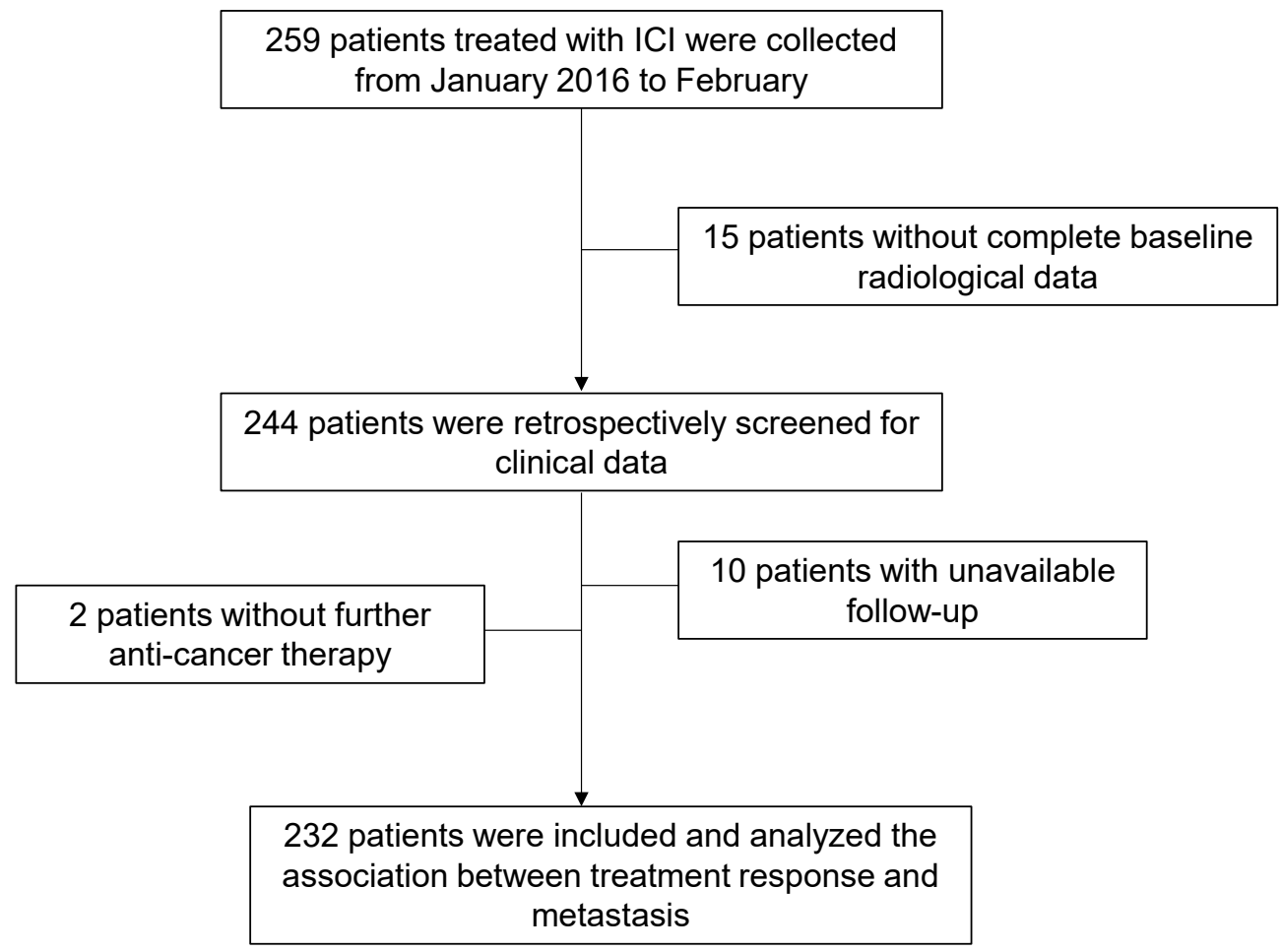

Figure S1 Flow chart of patients' selection.

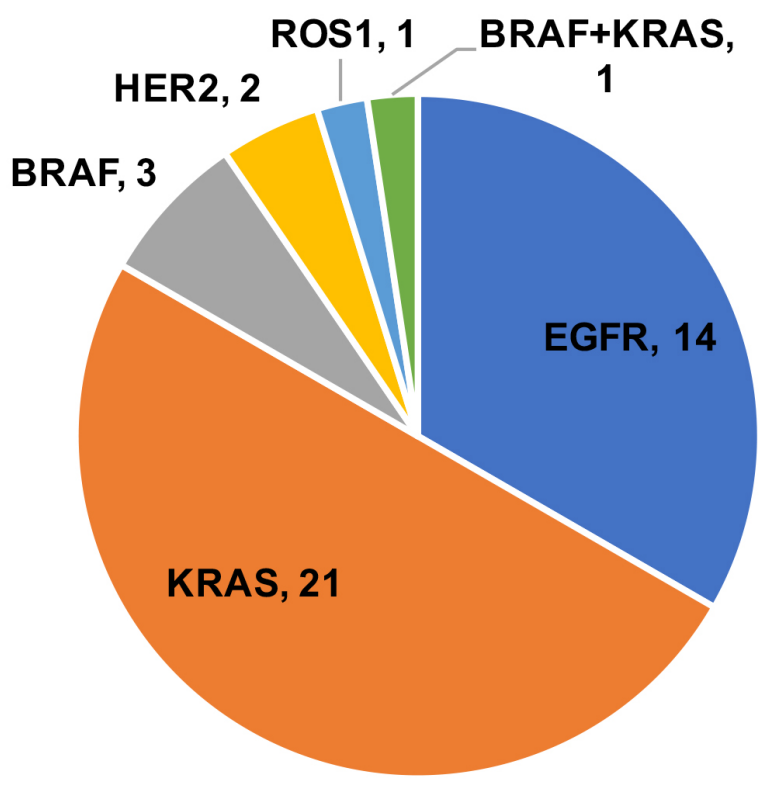

Figure S2 Mutations-testing results of 42 patients with genetic aberrations. 


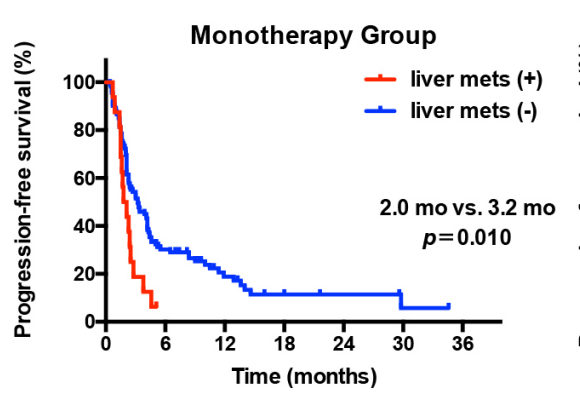

Combination Group
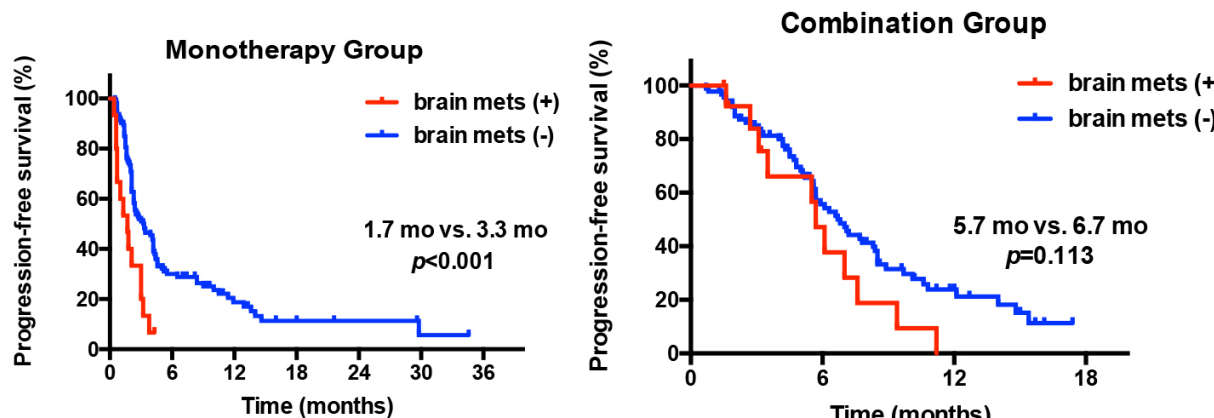

Contralaterallung metastasis
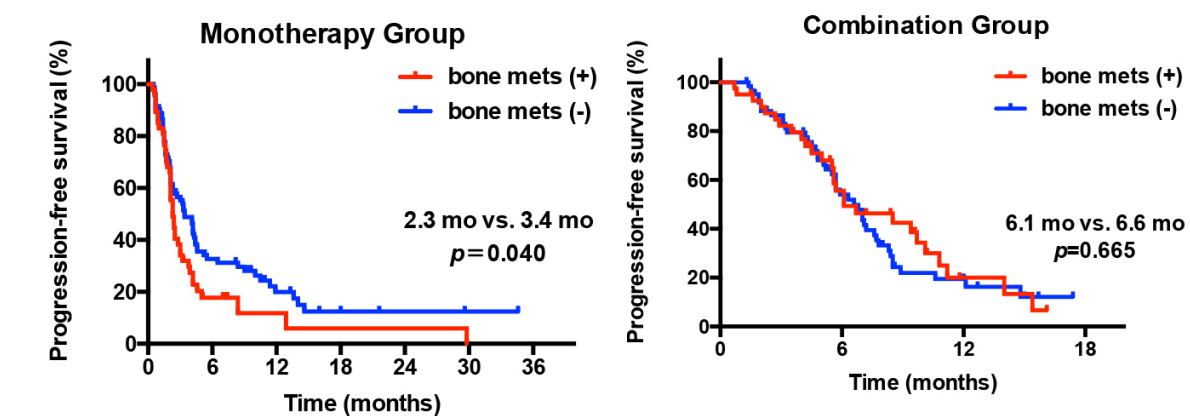

Pleural metastasis

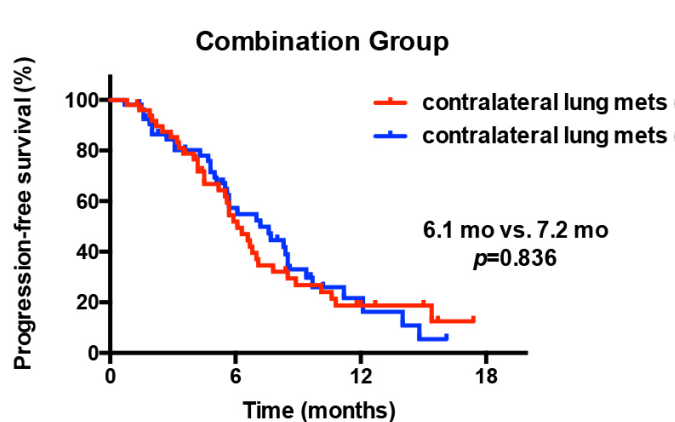

F
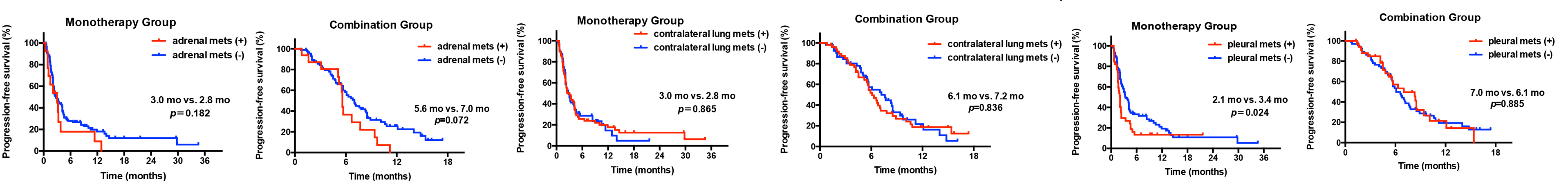

Time (months)

Time (months)

Figure S3 PFS in patients treated with ICI stratified by metastatic pattern. (A) Liver metastasis; (B) brain metastasis; (C) bone metastasis; (D) adrenal metastasis; (E) contralateral lung metastasis; (F) pleural metastasis. ICI, immune checkpoint inhibitor.

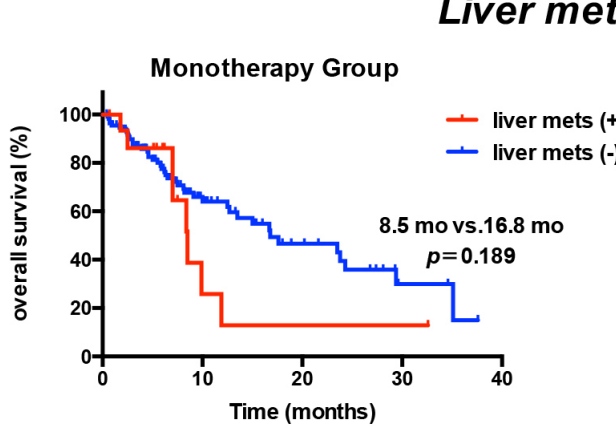

Liver metastasis

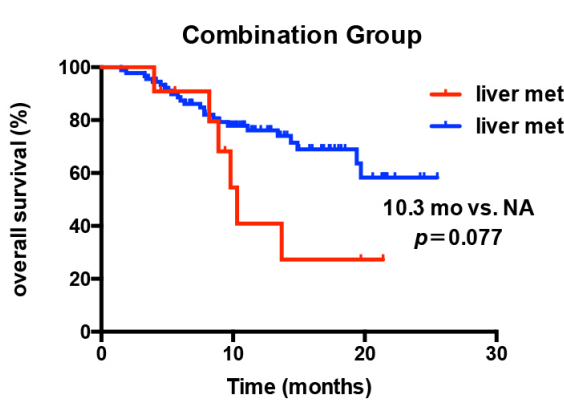

Adrenal metastasis

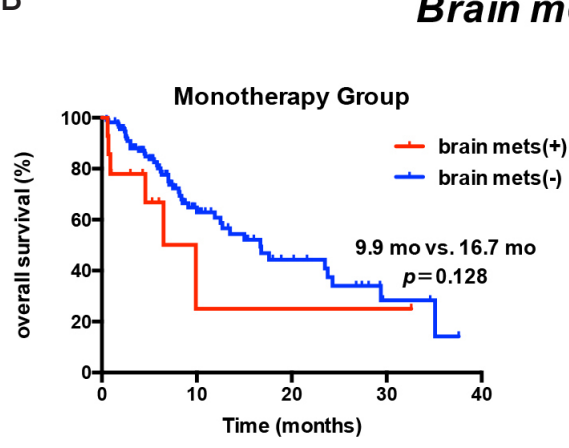

Brain metastasis

c

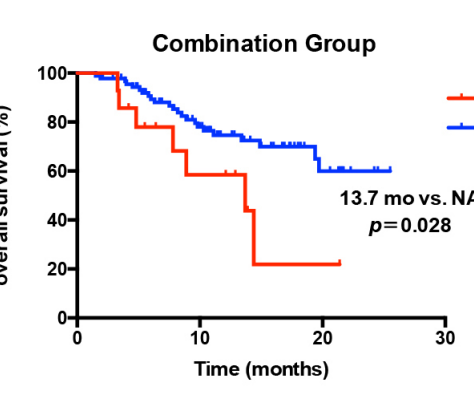

Contralaterallung metastasis
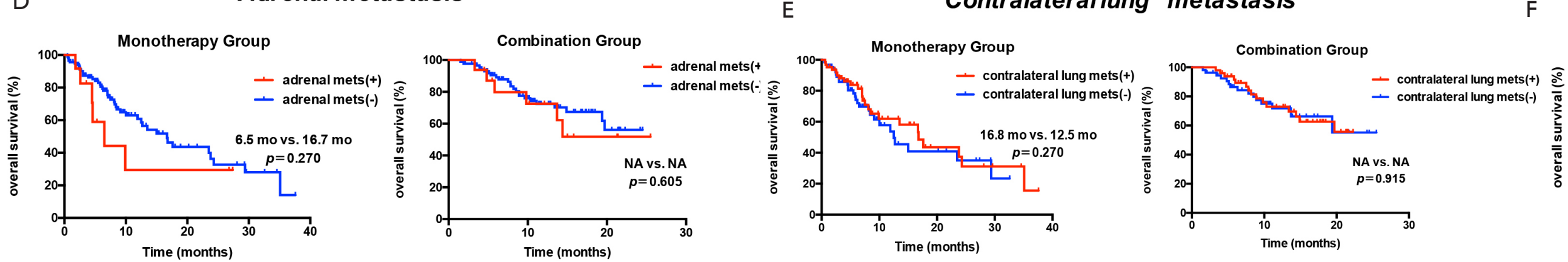
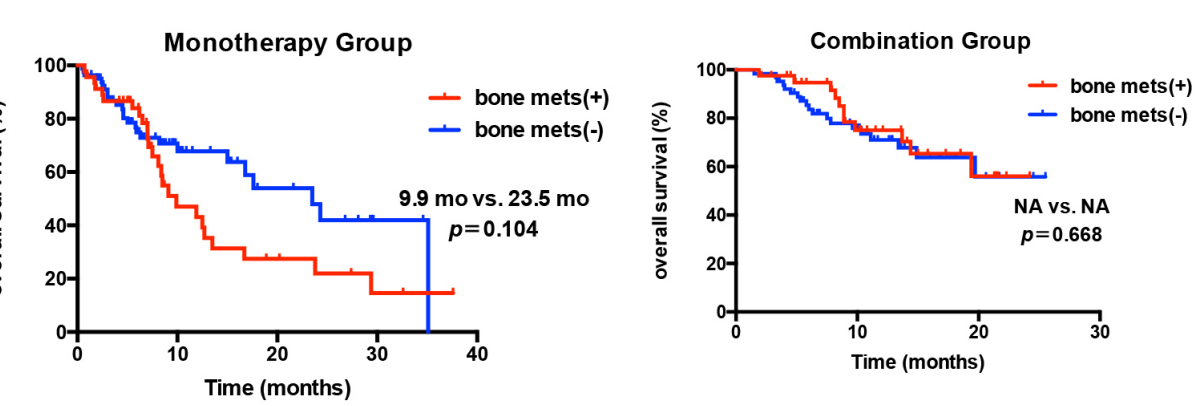

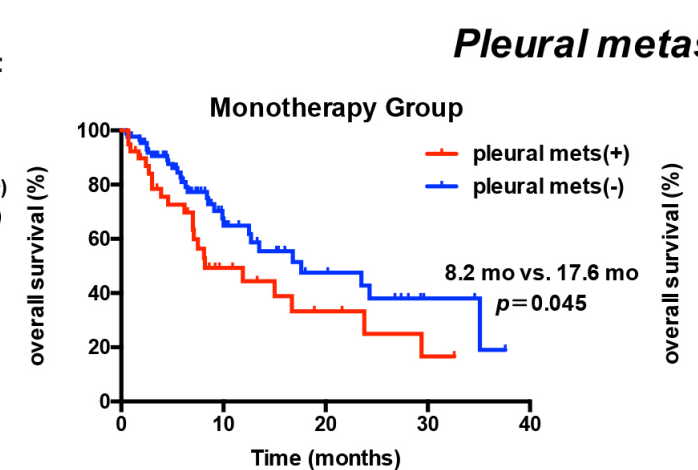

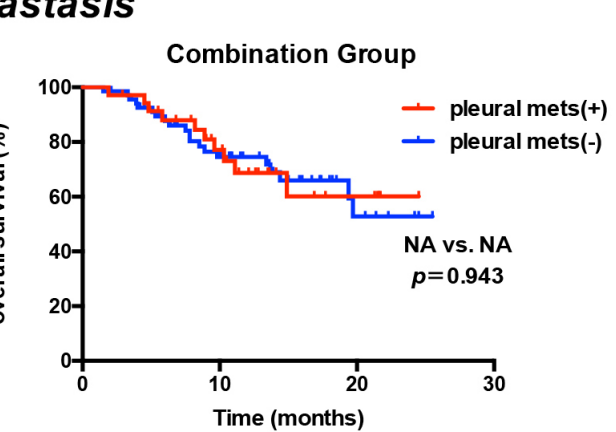

Figure S4 OS in patients treated with ICI stratified by metastatic pattern. (A) Liver metastasis; (B) brain metastasis; (C) bone metastasis; (D) adrenal metastasis; (E) contralateral lung metastasis; (F) pleural metastasis. ICI, immune checkpoint inhibitor. 
A

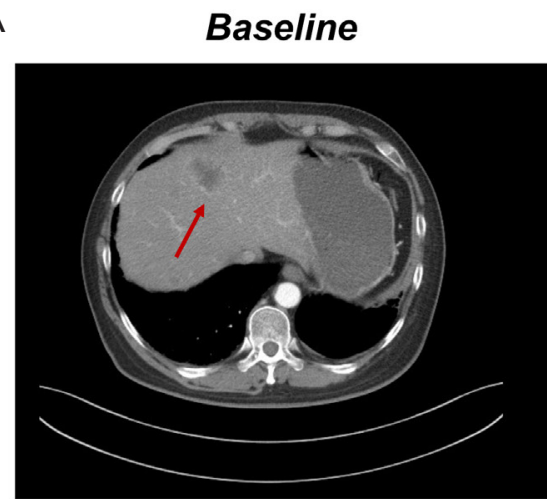

B

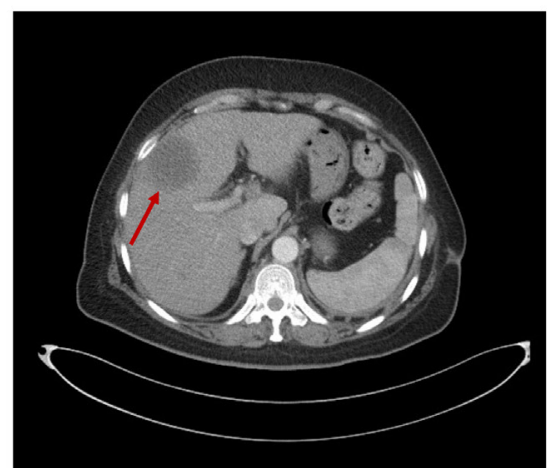

6 weeks of Pembrolizumab

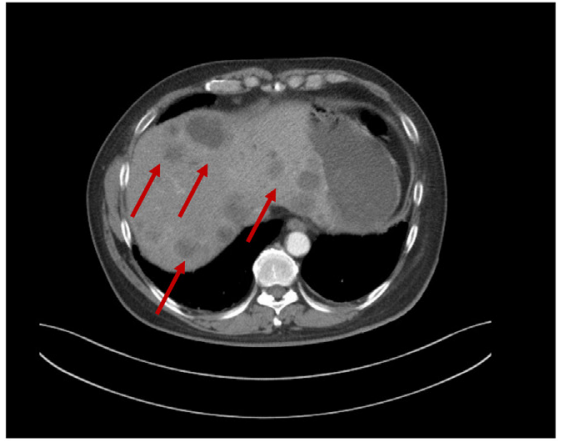

\section{6 weeks of Pembrolizumab+Chemotherapy}

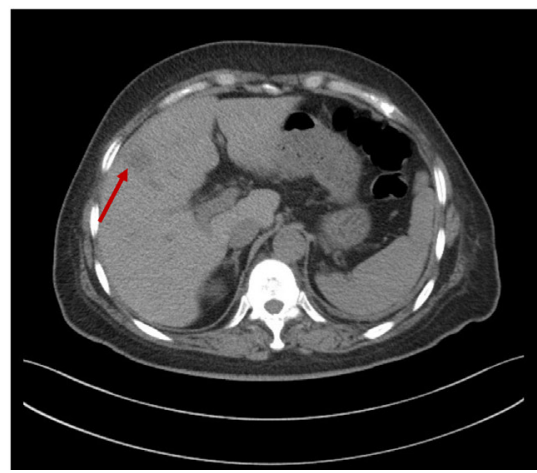

Figure S5 Representative intrahepatic response in patients with liver metastasis treated with ICI monotherapy or ICI combination therapy. (A) This male patient was diagnosed as stage IV lung squamous in March, 2017. After 4 cycles of chemotherapy, pulmonary lesion progressed. Therefore, the patient received pembrolizumab monotherapy as second-line treatment strategy. However, after two cycles of pembrolizumab, LM progressed but SD in pulmonary lesion was maintained; PFS was only 2.1 months. (B) This female patient was diagnosed as stage IV lung adenocarcinoma in January, Year 2019. This patient received combination therapy (pembrolizumab + pemetrexed + carboplatin) as first-line therapy. After two cycles of combination treatment, the patient exhibited partial response, with obviously shrunken hepatic lesions. At data cut-off, she was continuing to receive combination therapy with ongoing response. ICI, immune checkpoint inhibitor.

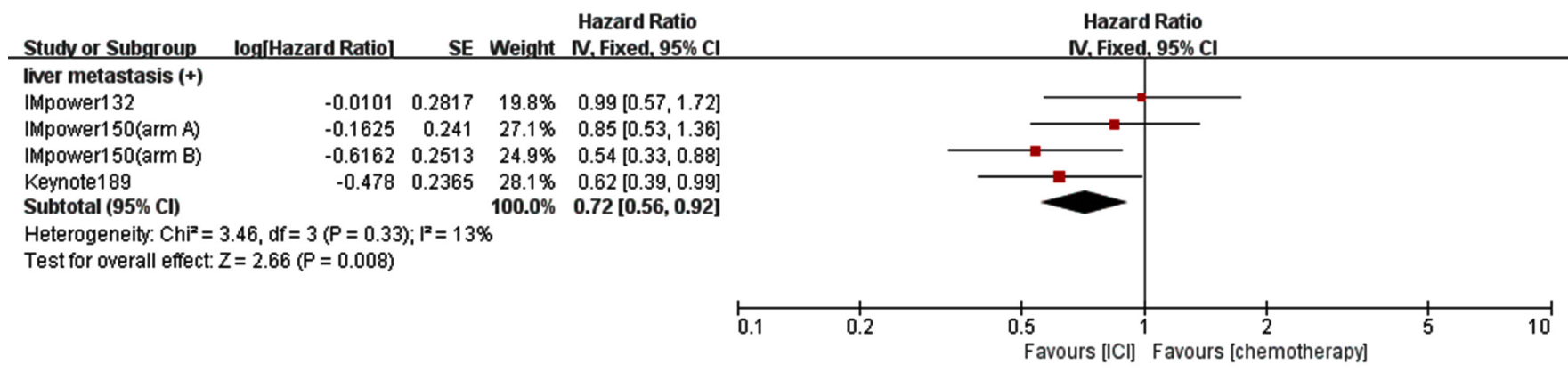

Figure S6 Meta-analysis of trials with available subgroup data on LM. LM, liver metastasis. 
Table S1 Lists of treatment ICI treatment strategies

\begin{tabular}{|c|c|c|}
\hline $\mathrm{ICl}$ & Combining strategy & No. of patients \\
\hline Nivolumab & - & 33 \\
\hline Pembrolizumab & - & 71 \\
\hline Atezolizumab & - & 1 \\
\hline IBI308 & - & 2 \\
\hline \multicolumn{3}{|c|}{$\mathrm{ICI}+$ chemotherapy } \\
\hline \multirow[t]{5}{*}{ Pembrolizumab } & Pemetrexed & 1 \\
\hline & Pemetrexed + carboplatin & 9 \\
\hline & Abraxane & 7 \\
\hline & Abraxane + carboplatin & 3 \\
\hline & Paclitaxel + carboplatin & 1 \\
\hline \multirow[t]{3}{*}{ Nivolumab } & Pemetrexed + carboplatin & 1 \\
\hline & Gemcitabine & 1 \\
\hline & Abraxane & 2 \\
\hline SHR-1210 & Pemetrexed + carboplatin & 30 \\
\hline JS001 & Pemetrexed + carboplatin & 5 \\
\hline
\end{tabular}

$\mathrm{ICl}$, immune checkpoint inhibitor. 
Table S2 Patient characteristics according to LM status

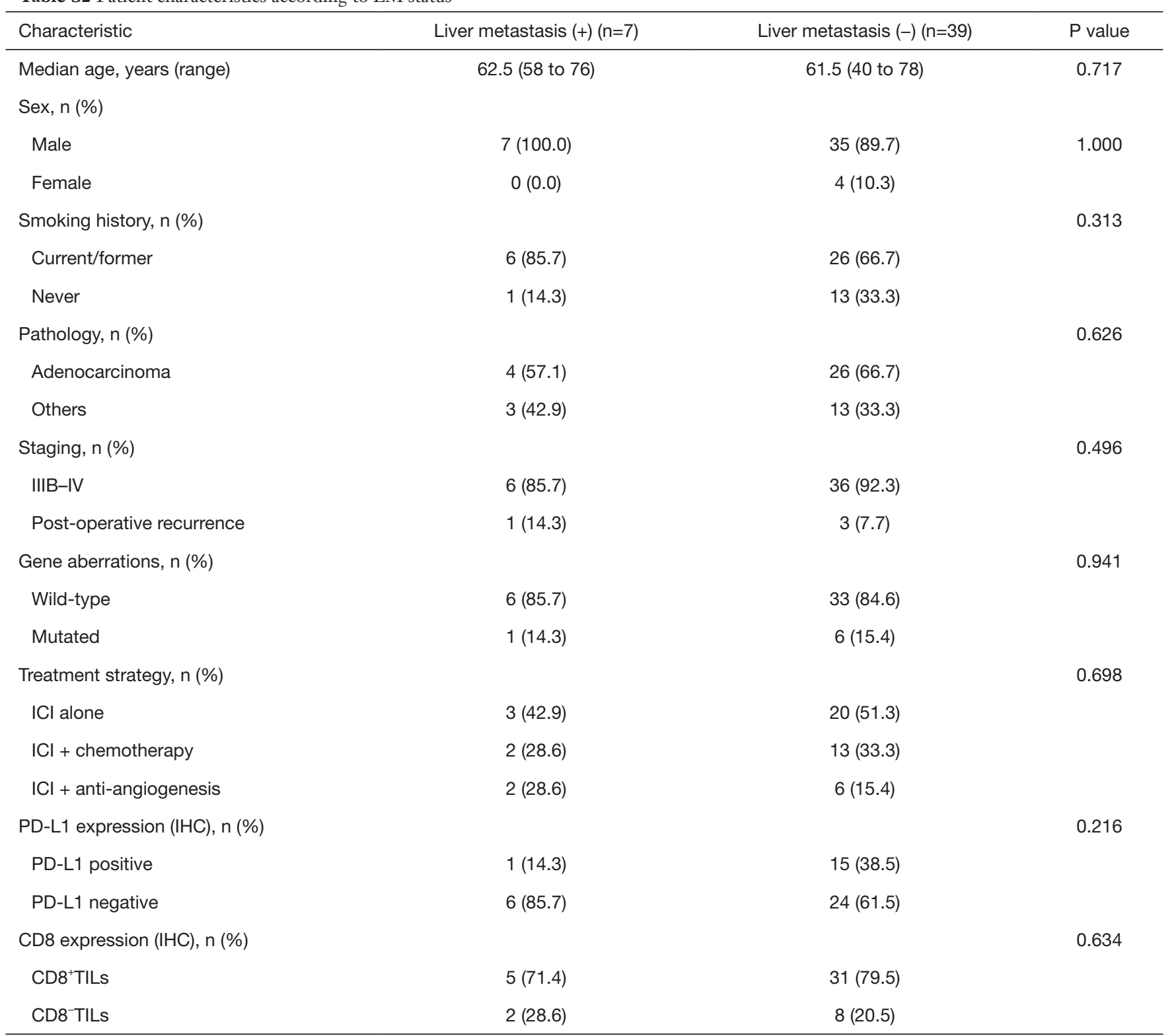

IHC, immunohistochemistry. 